\title{
Vertical instability with transient characteristics in KEK-Photon Factory electron storage ring
}

\author{
A. Mochihashi* \\ Research Center for Nuclear Physics (RCNP), Osaka University, 10-1 Mihogaoka, Ibaraki, Osaka, 567-0047 Japan \\ T. Obina, Y. Tanimoto, and T. Kasuga \\ Institute of Materials Structure Science, High Energy Accelerator Research Organization (KEK), 1-1 Oho, \\ Tsukuba, Ibaraki, 305-0801 Japan \\ (Received 11 August 2000; published 22 February 2001)
}

\begin{abstract}
A vertical instability of the KEK-Photon Factory electron storage ring was observed in a multibunch mode with empty buckets (bunch gap) using a bunch-by-bunch beam diagnostic system that consisted of a high-speed light shutter and an optical betatron oscillation detector. It was clearly seen that vertical betatron frequencies of individual bunches varied along a bunch train. The dependence of the betatron frequencies on the bunch positions in the train was explained by modulation of trapped-ion density caused by passage of the beam. Agreement between experimental results and the theoretical prediction was quite good.
\end{abstract}

DOI: 10.1103/PhysRevSTAB.4.022802

PACS numbers: 29.20.Dh, 29.27.Bd, 29.27.Fh

\section{INTRODUCTION}

In the Photon Factory (PF) electron storage ring, a vertical instability has been observed in a multibunch mode, in which a series of bunches followed by a series of empty buckets (bunch gap) is stored in the ring. The instability can be suppressed by exciting octupole magnets in routine operation for users; however, the origin of the phenomenon has not been perfectly understood yet. We have developed a bunch-by-bunch beam diagnostic system which can select a synchrotron radiation pulse only from a particular bunch in a bunch train and detect spatial oscillation of the picked-out pulse. Using the system, we have observed the betatron oscillation of individual bunches in the bunch train, and found that the betatron frequencies (tunes) of bunches depended on their positions in the train [1]. A possible cause of the phenomenon seems to be a transient ion related effect, such as the fast beam-ion instability (FBII) [2]; however, the measured bunch position dependence of the vertical tune was much larger than that predicted by the theory of FBII. In order to explain the phenomenon, we developed a model that shows that density of trapped ions is modulated by passage of the bunch train. Since trapped ions at a certain location in the ring are affected by a periodic focusing force due to passage of the bunch train, the ion density becomes time dependent. The dependence was calculated using a method similar to the theory of the betatron oscillation in circular accelerators, and the tune shifts of individual bunches were estimated. Agreement between experimental results and the theoretical prediction was good.

The bunch-by-bunch beam diagnostic system used in this study and the experimental results are described in

*Electronic address: mochi@ post.kek.jp
TABLE I. Main parameters of the PF ring.

\begin{tabular}{lcc}
\hline \hline \multicolumn{1}{c}{ Parameter } & Symbol & Value \\
\hline Beam energy & $E$ & $2.5 \mathrm{GeV}$ \\
rf frequency & $f_{\mathrm{rf}}$ & $500.1 \mathrm{MHz}$ \\
Revolution period & $t_{\mathrm{rev}}$ & $0.624 \mu \mathrm{sec}$ \\
Harmonic number & $h$ & 312 \\
Betatron tune & $\nu_{x} / \nu_{y}$ & $9.62 / 4.28$ \\
\hline \hline
\end{tabular}

Sec. II. The theoretical model which gives the tune dependence caused by modulation of the ion density is given in Sec. III. Discussions of the experimental results with the theory are given in Sec. IV.

Main parameters of the PF [3] are shown in Table I. The symbols in the table will be used without further notice in this paper.

\section{EXPERIMENTS}

\section{A. Bunch-by-bunch beam diagnostic system}

In order to select a synchrotron radiation pulse only from a particular bunch in a bunch train, we have developed a high-speed light shutter [4]. A schematic diagram of the shutter is shown in Fig. 1. A pockels cell (Fastpulse Technology, 1044-FW) is placed between polarization filters whose polarization angles are perpendicular to each other. The incident light can pass through the shutter while a high voltage pulse is applied to the cell because it rotates the polarization plane. Since the time response of the cell is fast enough, the operation speed of the shutter is mainly determined by the rise/fall time of the pulser. A high voltage pulser generates pulses with a width (FWHM) of about $1.5 \mathrm{~ns}$, which is shorter than the bunch spacing of $2 \mathrm{~ns}$ and a height of $550 \mathrm{~V}$. We operate the shutter with a repetition rate of $f_{\text {shutter }}=534 \mathrm{kHz}$ which corresponds to one-third of the revolution frequency because of limitation of the 


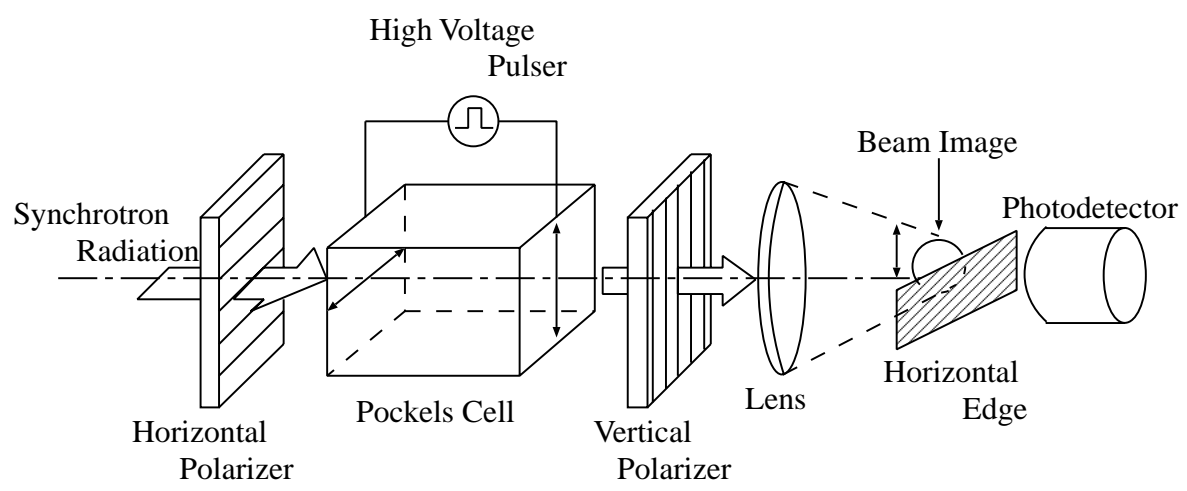

FIG. 1. Schematic diagram of the high-speed light shutter and the optical betatron oscillation detector.

repetition rate of the high voltage pulser (maximum $600 \mathrm{kHz}$ ) and the reason described below.

Light through the shutter is focused by a lens to form an image of the beam on a horizontal edge that cuts off half of the image. Intensity of light through the edge is detected by a photomultiplier tube (PMT, Hamamatsu Photonics, H2431-50). Vertical motion of the beam can be detected as amplitude variation of the output signal of the PMT. The output signal of the PMT is analyzed with a spectrum analyzer (ADVANTEST, R3361D).

An extinction ratio of the shutter, which is defined as a ratio of intensity of the picked-out light to that of the leaked one, was 300 at most. Therefore, the contribution of leaked light pulses through the shutter during the closing time is not negligible. The contribution of the leaked signal corresponding to all bunches of the spectrum appears on both sides of the harmonics of the revolution frequency. On the other hand, spectral lines corresponding to the selected bunch appear on both sides of the harmonics of the shutter frequency $f_{\text {shutter }}$. Therefore, we can distinguish the betatron oscillation of the selected bunch from the contribution of the other bunches by detecting a betatron sideband at a frequency $f_{\text {obs }}$ that is not a sideband of a harmonic of the revolution frequency $f_{\text {rev }}$, but of a harmonic of a shutter frequency $\left(f_{\text {shutter }}=\frac{1}{3} f_{\text {rev }}\right)$, i.e.,

$$
f_{\text {obs }}=\frac{n}{3} f_{\text {rev }} \pm q f_{\text {rev }} \quad\left(\frac{n}{3} \neq \text { integer }\right),
$$

where $q$ is the decimal part of the vertical tune $(0.28$ in the PF).

It is a merit that the light shutter is free from harmful effects caused by ringing that commonly occurs in fast electronic switches, and excellent tolerance to electronic noise is another advantage of the optical system. On the other hand, it is a demerit that the system is weak in quantitative detection of the betatron oscillation amplitude.

\section{B. Tune measurement}

Betatron oscillations of all bunches due to the instability are detected independently in several multibunch conditions shown in Table II. In order to make the obser- vation of the instability clear, we turned off the octupole magnets to avoid the Landau damping effect during the experiments. Therefore, amplitude-dependent tune shift due to the magnetic system is negligible. An initial bunch current was set to be $1.6 \mathrm{~mA} /$ bunch in all experiments. Figures 2 and 3 show spectra which correspond to the betatron sidebands of the 1st and 20th bunches in the 280 bunch train, respectively. Although the beam currents of these two bunches were almost the same, not only these spectral powers but also these frequencies are different as seen in the figures.

In order to determine the tunes, we calculated the first order moments of the spectra. Figures 4-6 show measured

TABLE II. Experimental conditions. (The bunch current is $1.6 \mathrm{~mA} /$ bunch in all experiments.)

\begin{tabular}{cccc}
\hline \hline Experiments & $\begin{array}{c}\text { Number of } \\
\text { bunches }\end{array}$ & $\begin{array}{c}\text { Empty } \\
\text { buckets }\end{array}$ & $\begin{array}{c}\text { Change in tune } \\
\left(\frac{\Delta \nu_{y}}{\Delta n}\right) \\
\left(\times 10^{-6} / \text { bunch }\right)\end{array}$ \\
\hline Exp. 1 & 280 & 32 & 4.0 \\
Exp. 2 & 100 & 212 & 2.1 \\
Exp. 3 & 50 & 262 & 1.7 \\
\hline \hline
\end{tabular}

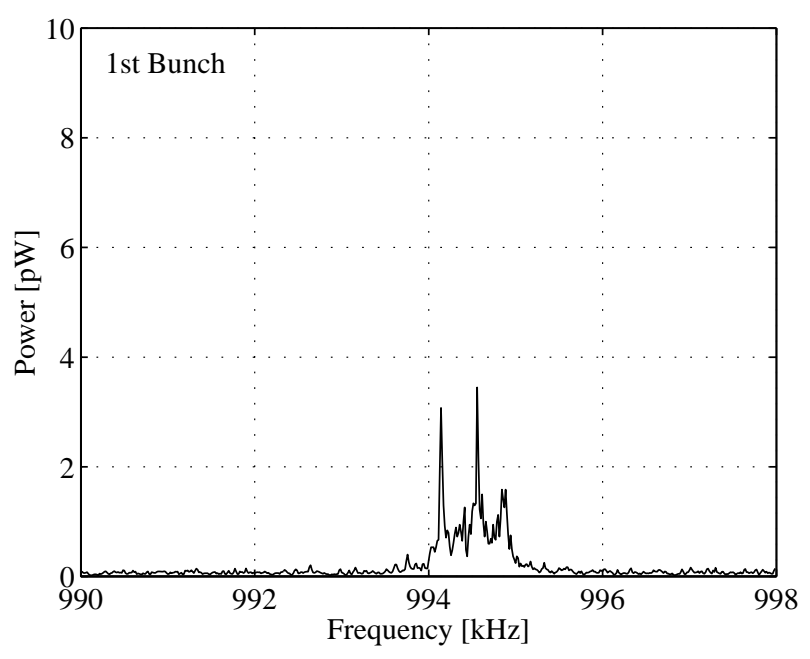

FIG. 2. Spectrum of the 1 st bunch in the bunch train at $f_{\text {shutter }}+q f_{\text {rev }}$. 


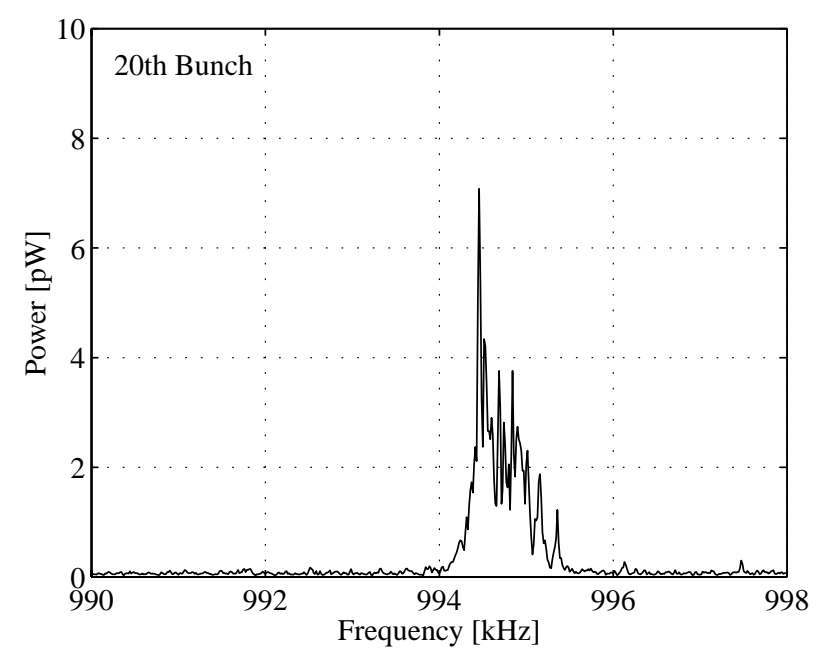

FIG. 3. Spectrum of the 20th bunch in the bunch train at $f_{\text {shutter }}+q f_{\text {rev }}$.

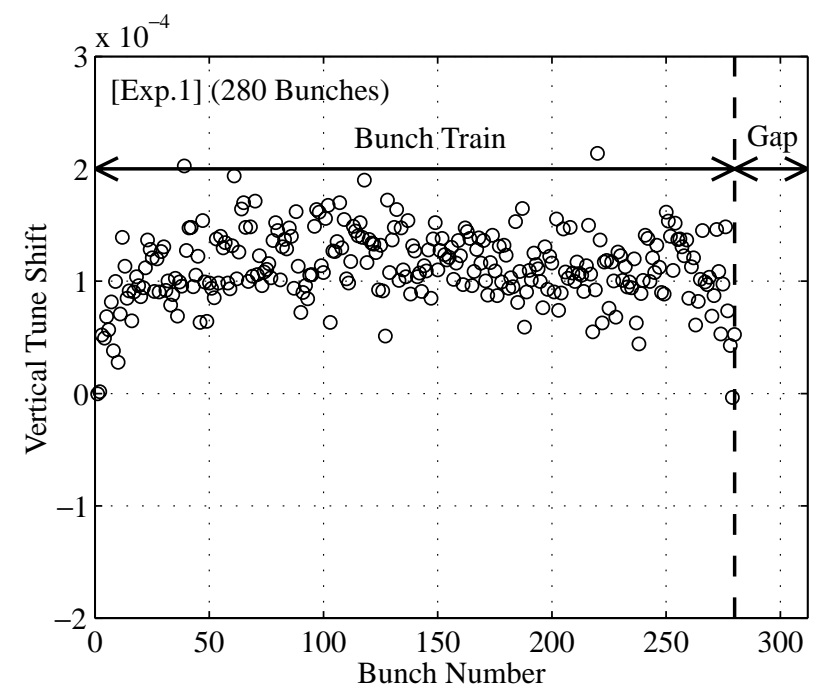

FIG. 4. Vertical tune shifts of individual bunches along the bunch train with 280 bunches.

tune shifts from the tune of the first bunch as a function of the bunch position in the train. Because the bunch current varies during the measurement, it is necessary to correct the tune which depends on the beam current. In order to obtain data for the correction, we measured the dependence of the tune on the beam current in a single-bunch mode, in which the ion-trapping effect does not occur. The measured current dependence of the tune is $1.2 \times 10^{-4} / \mathrm{mA}$, therefore the correction is not large compared with the measured tune shift. Furthermore, we controlled the initial bunch currents of individual bunches within $10 \%$ to avoid the current dependence of the tune. When the train length is long ( 280 bunches), it is clearly seen that the tunes gradually increase along the train in the head and decrease again in the tail as seen in Fig. 4.

Changes in the vertical tunes $\left(\frac{\Delta \nu_{y}}{\Delta n}\right)$ along the successive 20 bunches in the head of the train are evaluated by linear

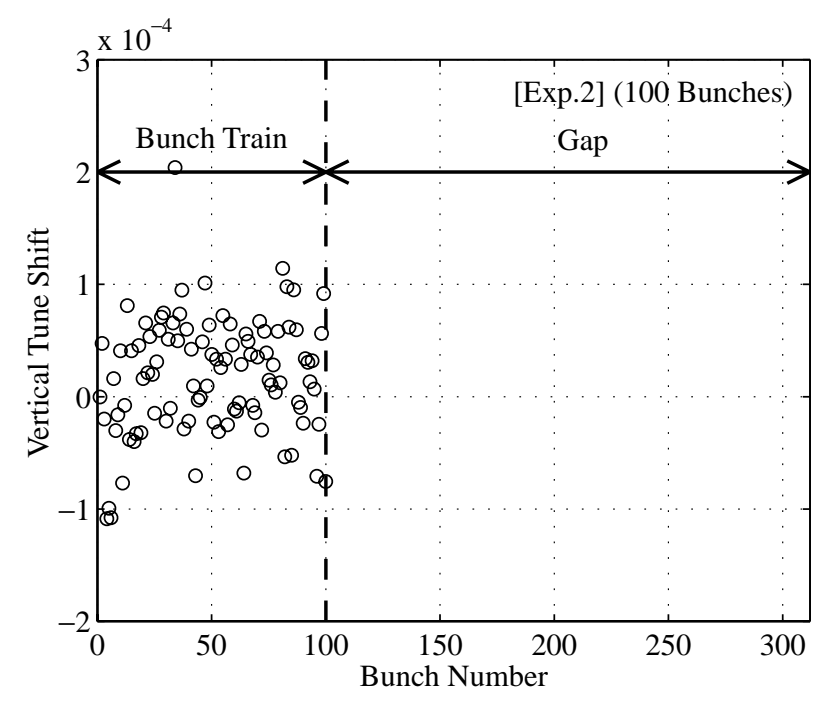

FIG. 5. Vertical tune shifts of individual bunches along the bunch train with 100 bunches.

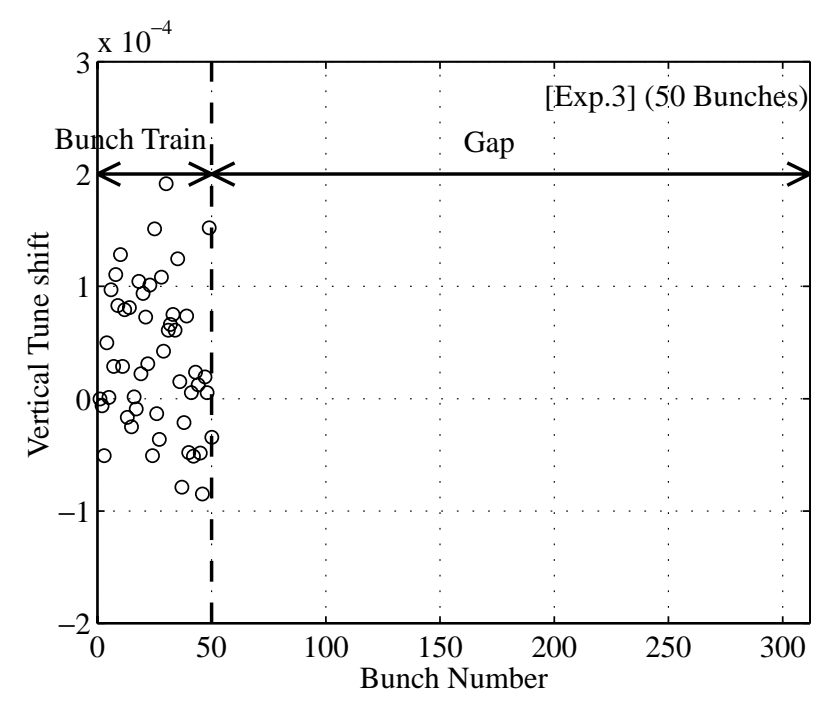

FIG. 6. Vertical tune shifts of individual bunches along the bunch train with 50 bunches.

fitting, and shown in Table II. The change in the vertical tune along the bunch train caused by FBII is estimated at $\frac{\Delta \nu_{y}}{\Delta n}=1.8 \times 10^{-7} /$ bunch at the same condition shown in Table II. The results seem to be related to the fact that the bunch gap with 32 empty buckets is not sufficient to clear ions completely, therefore the electron beam is surrounded by an ion cloud at this condition.

\section{THEORY}

Because ions at a certain location in the storage ring are affected by a periodic focusing force with a period of $t_{\text {rev }}$ corresponding to a configuration of the bunch train, ion motion can be discussed with a method similar to the theory of the betatron oscillation in circular accelerators. Namely, an "ion betatron function $\beta_{i}$ " with the period $t_{\mathrm{rev}}$ 


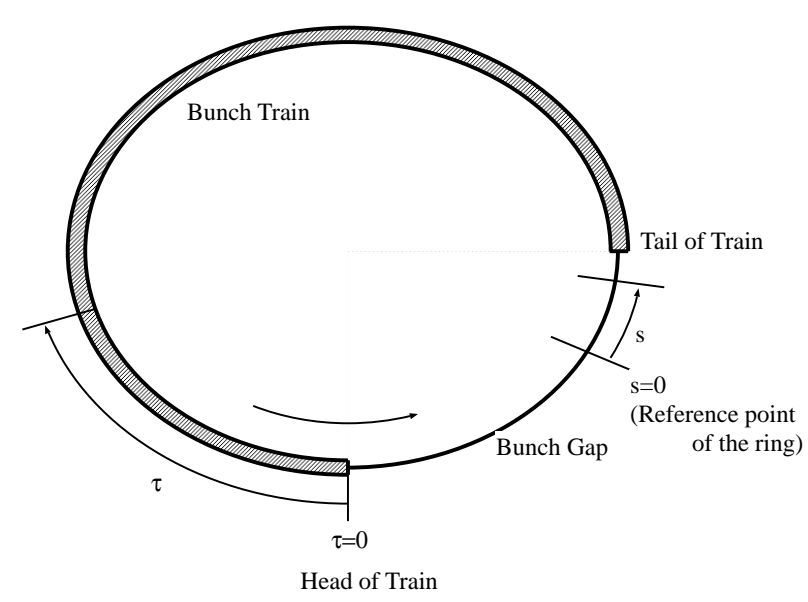

FIG. 7. Definition of parameters $s$ and $\tau$.

can be defined as a function of the time lapse $\tau$ from passage of the bunch head. Vertical displacement $y_{i}$ of an ion at time $t$ is given by

$$
y_{i}(t)=y_{0} \sqrt{\beta_{i}(\tau)} \cos \phi(t),
$$

where $y_{0}$ is a constant and $\phi(t)$ is a phase of the ion motion. Figure 7 shows definitions of $\tau$ and the location $s$ in the ring.

Because trapped ions oscillate around the electron beam with amplitudes proportional to $\sqrt{\beta_{i}}$, the size of the ion cloud is proportional to $\sqrt{\beta_{i}}$; and because $\sqrt{\beta_{i}}$ is a periodic function of $\tau$, density of the ions is a function of $\tau$ with a period of $t_{\text {rev }}$. According to the "classical" theory of the ion trapping [5,6], the trapped ions cause tune shifts proportional to their density. Therefore, the variation of the ion density could cause the tune shifts of bunches that depend on the positions in the train.

\section{A. Ion motion}

Vertical displacement of an ion affected by the bunch train can be expressed by a matrix form [6]:

$$
\begin{aligned}
\left(\begin{array}{c}
y_{i}\left(t+t_{\mathrm{rf}}\right) \\
\dot{y}_{i}\left(t+t_{\mathrm{rf}}\right)
\end{array}\right) & =\left(\begin{array}{cc}
1 & t_{\mathrm{rf}} \\
0 & 1
\end{array}\right)\left(\begin{array}{cc}
1 & 0 \\
a & 1
\end{array}\right)\left(\begin{array}{l}
y_{i}(t) \\
\dot{y}_{i}(t)
\end{array}\right) \\
& =D B\left(\begin{array}{c}
y_{i}(t) \\
\dot{y}_{i}(t)
\end{array}\right),
\end{aligned}
$$

where $t_{\mathrm{rf}}$ is the bunch spacing $\left(=1 / f_{\mathrm{rf}}\right), a$ denotes the impulse due to passage of one bunch:

$$
a=-\frac{2 r_{p} c N_{b}}{A \sigma_{y}\left(\sigma_{x}+\sigma_{y}\right)},
$$

where $r_{p}$ is the classical proton radius, $c$ the speed of light, $N_{b}$ the number of the electrons in the bunch, $A$ the mass number of the ion, and $\sigma_{x}, \sigma_{y}$ the horizontal and vertical beam sizes, respectively.

In Eq. (3), the matrices $B$ and $D$ represent a thin-lens kick by a bunch and a drift between the bunches, respec- tively. The matrix for passage of the whole bunch train that consists of bunches with a same beam current can be written by multiplication of $D B$ and $D$, namely,

$$
\begin{aligned}
\left(\begin{array}{c}
y_{i}\left(t+t_{\mathrm{rev}}\right) \\
\dot{y}_{i}\left(t+t_{\mathrm{rev}}\right)
\end{array}\right) & =D^{h-n_{b}}(D B)^{n_{b}}\left(\begin{array}{l}
y_{i}(t) \\
\dot{y}_{i}(t)
\end{array}\right) \\
& =M\left(\begin{array}{c}
y_{i}(t) \\
\dot{y}_{i}(t)
\end{array}\right)
\end{aligned}
$$

where $n_{b}$ is the number of bunches in the train.

\section{B. "Ion Twiss parameters"}

Because the ions are affected by the periodic focusing force corresponding to a configuration of the bunch train, "ion Twiss parameters" can be defined as a function of the time lapse $\tau$ from passage of the bunch head. The parameters at the $n$th bunch in the train are written by

$$
\left(\begin{array}{c}
\beta_{i}\left(n t_{\mathrm{rf}}\right) \\
\alpha_{i}\left(n t_{\mathrm{rf}}\right) \\
\gamma_{i}\left(n t_{\mathrm{rf}}\right)
\end{array}\right)=N_{\text {bunch }}^{n}\left(\begin{array}{c}
\beta_{i}(0) \\
\alpha_{i}(0) \\
\gamma_{i}(0)
\end{array}\right),
$$

where $\beta_{i}(0), \alpha_{i}(0)$, and $\gamma_{i}(0)$ are the parameters at the head of the bunch train, $N_{\text {bunch }}$ is the $3 \times 3$ transformation matrix of these parameters composed of matrix elements of $D B$ [7]. Because the bunch train has a mirror symmetry around the center of the train, the betatron function of the ion $\beta_{i}(\tau)$ is also mirror symmetric. Examples of $\beta_{i}$ for the same conditions in Exp. 1 at two different locations in the ring are shown in Fig. 8. In the calculation of $\beta_{i}$, the $\mathrm{CO}^{+}$ion which is a main component of the residual gas ion species in the PF is assumed.

\section{Tune shifts due to trapped ions}

In the classical theory of the ion trapping, the size of the ion cloud is assumed to be equal to that of the beam. However, as discussed in the previous section, the size

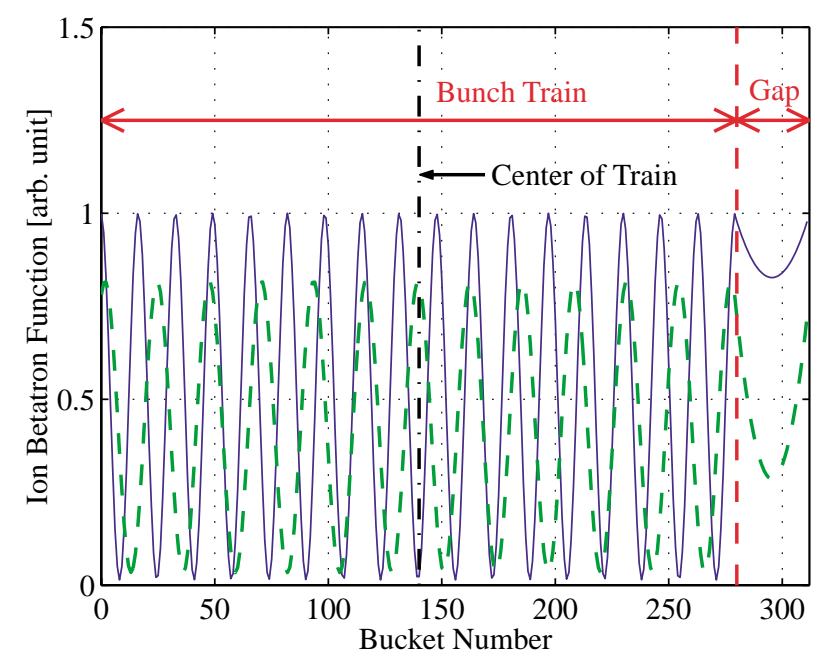

FIG. 8. (Color) Ion betatron functions (solid-blue and dashedgreen curves) at two different locations in the ring. 
of the ion cloud is modulated by passage of the bunch train. Considering the effect of the modulation, we assume that the horizontal/vertical size $\Sigma_{x, y}(\tau)$ of the ion cloud is proportional to the horizontal/vertical beam sizes $\sigma_{x, y}$ and time dependent; namely,

$$
\Sigma_{x, y}(\tau)=\epsilon_{x, y}(\tau) \sigma_{x, y},
$$

where $\epsilon_{x, y}(\tau)$ is a factor which gives a time dependence but is independent of $s$.

If the ion cloud is assumed to be a Gaussian distribution, a vertical component of an electric field $\mathcal{F}_{y}$ induced by the cloud under linear approximation is

$$
\mathcal{E}_{y}(\tau)=\frac{e}{2 \pi \epsilon_{0}} \frac{\eta \lambda_{e}}{\Sigma_{y}(\tau)\left[\Sigma_{x}(\tau)+\Sigma_{y}(\tau)\right]} y,
$$

where $\lambda_{e}$ is the averaged line density of the electrons, $\eta$ the neutralization factor, $e$ the electron charge, and $\epsilon_{0}$ the dielectric constant of the free space.

Because the beam size $\sigma_{x, y}$ depends on the location $s$ in the storage ring, the electric field $\mathcal{E}_{y}$ also depends on $s$. From Eq. (8), a vertical tune shift $\Delta \nu_{y}(\tau)$ caused by the trapped ions is written by

$$
\begin{aligned}
\Delta \nu_{y}(\tau) & =\frac{1}{4 \pi} \int_{C} \frac{e}{E} \frac{\partial \mathcal{E}_{y}(\tau)}{\partial y} \beta_{y} d s \\
& =\frac{r_{e} E_{0}}{2 \pi E} \lambda_{e} \eta \int_{C} \frac{\beta_{y}}{\Sigma_{y}(\tau)\left[\Sigma_{x}(\tau)+\Sigma_{y}(\tau)\right]} d s,
\end{aligned}
$$

where $E_{0}$ is the rest mass of an electron, $r_{e}$ the classical electron radius, and $\beta_{y}$ the betatron function of the beam, not the function for the ions. The integral in Eq. (9) is taken over the area $C$ where ions are stably trapped around the beam.

Now we rewrite Eq. (9) using Eq. (7):

$$
\begin{aligned}
\Delta \nu_{y}(\tau) & =\frac{r_{e} E_{0}}{2 \pi E} \frac{\lambda_{e} \eta}{\epsilon_{x}(\tau) \epsilon_{y}(\tau)} \int_{C} \frac{\beta_{y}}{\sigma_{y}\left(\sigma_{x}+\frac{\epsilon_{y}(\tau)}{\epsilon_{x}(\tau)} \sigma_{y}\right)} d s \\
& \approx \frac{\Delta \nu_{y}^{0}}{\epsilon_{x}(\tau) \epsilon_{y}(\tau)}
\end{aligned}
$$

where $\Delta \nu_{y}^{0}$ is the tune shift in the classical theory of the ion trapping:

$$
\Delta \nu_{y}^{0}=\frac{r_{e} E_{0}}{2 \pi E} \lambda_{e} \eta \int_{C} \frac{\beta_{y}}{\sigma_{y}\left(\sigma_{x}+\sigma_{y}\right)} d s .
$$

Here, the horizontal motion of the ions was neglected because the horizontal beam size is larger than the vertical size. If we assume that the horizontal size of the ions is equal to the beam size $\left[\epsilon_{x}(\tau)=1\right]$, the tune shift along the bunch train is simply written by

$$
\Delta \nu_{y}(\tau)=\frac{\Delta \nu_{y}^{0}}{\epsilon_{y}(\tau)}
$$

Because the impulse acting on the ions depends on the beam size where the ions are trapped, $\beta_{i}(\tau)$ depends on locations in the ring. The size of the ion cloud is propor- tional to $\sqrt{\beta_{i}(\tau)}$, and the ratio $1 / \epsilon_{y}(\tau)$ is also proportional to an average of $1 / \sqrt{\beta_{i}(\tau)}$ over the whole ring, namely,

$$
\frac{1}{\epsilon_{y}(\tau)}=b_{0} \int_{C} \frac{1}{\sqrt{\beta_{i}(\tau)}} d s
$$

where $b_{0}$ is a normalization constant which satisfies

$$
\frac{1}{n_{b} t_{\mathrm{rf}}} \int_{0}^{n_{b} t_{\mathrm{rf}}} \frac{1}{\epsilon_{y}(\tau)} d \tau=1
$$

We can estimate the tune shift along the bunch train due to the modulation of the ion density from Eq. (12) with Eq. (13).

\section{DISCUSSION}

We calculated the theoretical values of the tune shifts along the train taking into account the modulation of ion density using Eq. (12). Figure 9 shows the experimental and the theoretical values of the tune shifts of all bunches corresponding to Exp. 1. In the calculation of theoretical values, the ion species of $\mathrm{CO}^{+}$and the neutralization factor $\eta$ of $9.4 \times 10^{-6}$ are assumed. The ion betatron function $\beta_{i}$ is symmetric around the center of the bunch train in itself as mentioned above. Asymmetry seen in the calculated tune shift arises from the change in beam current during the measurement. In order to estimate $\eta$ independently, we measured the dependence of the tune on the total beam current keeping the pattern of the bunch train unchanged. Because the current dependence of the tune is small, the accuracy of the measurement is not sufficient, however, the estimated $\eta$ of $7.5 \times 10^{-5} \pm 6.9 \times 10^{-5}$ is consistent with the value assumed above.

The pattern of the calculated tune shift meanders in unison with the modulation of the ion density due to the oscillation of ions as seen in Fig. 9. Although amplitude and periods of wiggles in the middle part of the bunch train are

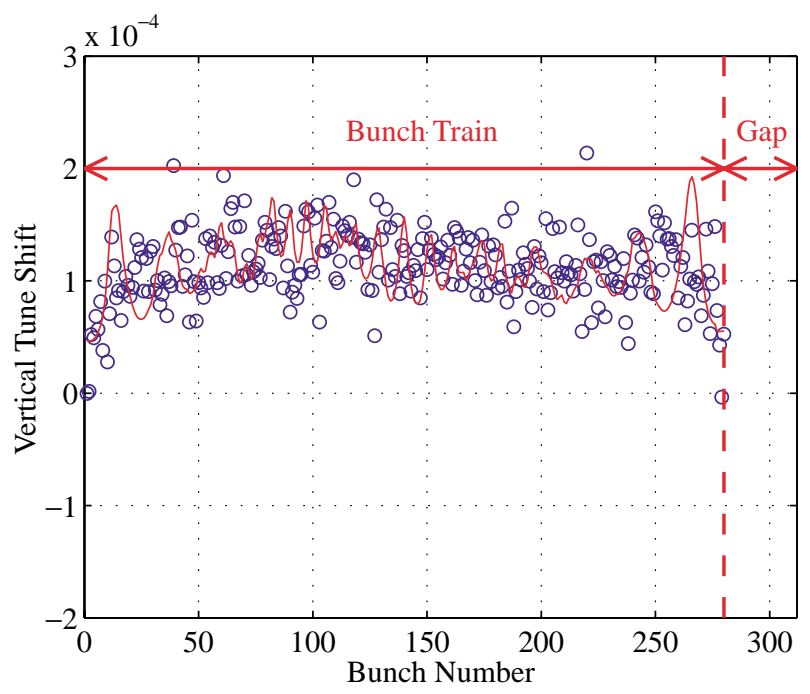

FIG. 9. (Color) The change of the tunes along the 280 bunch train. Blue circles and a red curve correspond to the experimental results and the theoretical values, respectively. 
dispersed by averaging Eq. (13), the pattern has a characteristic that it rapidly rises in the head of the train and falls in the tail. This characteristic is also clearly seen in the experimental results of Fig. 9. The increase of the tunes in the head of the bunch train is always observed in a series of experiments. As a measure of the phenomenon, we calculated the averaged tune shifts along 20 bunches in the head. The theoretical value of $\frac{\Delta \nu_{y}}{\Delta n}=5.1 \times 10^{-6} /$ bunch was obtained. Agreement between the experimental value shown in Table II and the theoretical value is fairly good.

The errors of the tune shift measurement are estimated at $\pm 0.5 \times 10^{-4}$ that is almost the same as an amplitude of a wiggle of the tune shift pattern in the middle part of the bunch train. However, the wiggle pattern seen in the calculation reappears in the measurement, although peaks and valleys of both patterns do not coincide with each other in the middle part of the train. The wiggle pattern of $\epsilon_{y}(\tau)$ in the middle part of the bunch train is smeared in the averaging of $1 / \sqrt{\beta_{i}}$ given in Eq. (13); however, the pattern of the tune shifts in the head and the tail remains in the averaging, because ion betatron functions at any location in the ring have a tendency to decrease along the bunch train head and increase along the tail. This is one of the reasons why the tune shift pattern in the middle part of the train was vague but had a clear increase in the head and a decrease in the tail.

In the case of the short bunch trains (Exps. 2 and 3), the variations of the tune shifts were small compared with the case of the long train (Exp. 1). According to the classical theory of the ion trapping, ions such as $\mathrm{CO}^{+}$cannot be trapped around the beam if the number of the bunches in the train is less than 100 in the PF. The experiments (Exps. 2 and 3) seem to be explained by FBII. However, the experimental values are roughly 10 times as large as that predicted by FBII. This fact suggests that not only FBII but also the effect proposed in Sec. III above affect the tune. Ion clouds can remain around the beam because of nonlinearity of the focusing force of the beam even though the number of bunches is less than 100 .

We built this optical beam diagnostic system on a beam line for a photon counting system that requires merely weak light, therefore, an intensity of light is insufficient and the $S / N$ ratio of measurement is unsatisfactory at present. Improvement of the beam line and development of an optical beam diagnostic system that is able to detect the oscillation amplitude quantitatively are future subjects.

\section{ACKNOWLEDGMENTS}

The authors express their sincere thanks to Professor M. Kobayashi and staffs of the light source division who have been kindly supporting our experiments. One of the authors (A. M.) thanks the Research Fellowships of the Japan Society for the Promotion of Science for Young Scientists for its financial support.

[1] T. Obina et al., in Proceedings of the 7th European Particle Accelerator Conference, Vienna, 2000, http.// accelconf.web.cern.ch/AccelConf/e00/index.html

[2] T. O. Raubenheimer and F. Zimmermann, Phys. Rev. E 52, 5487 (1995).

[3] Photon Factory Activity Report, 1998, edited by Y. Hori et al. (unpublished).

[4] A. Mochihashi et al., in Proceedings of the 1st Asian Particle Accelerator Conference, Tsukuba, 1998, edited by Y. H. Chin et al. (High Energy Accelerator Research Organization, Tsukuba, 1998), p. 549.

[5] R. D. Kohaupt, DESY Interner Bericht Report No. H171/2, 1971 (unpublished).

[6] Y. Baconnier and G. Brianti, CERN Internal Report No. CERN/SPS/80-2 (DI), 1980 (unpublished).

[7] H. Wiedemann, Particle Accelerator Physics (SpringerVerlag, Berlin, 1998). 